\title{
Skin Surface Protein Detection by Transdermal Analysis Patches in Pediatric Psoriasis
}

\author{
Mirjam J. Schaap ${ }^{a}$ Finola M. Bruins ${ }^{a} X_{\text {Xehui He }}^{b}$ Kadri Orroc, d \\ Malou Peppelman ${ }^{a}$ Piet E. J. van Erp ${ }^{a}$ Elke M. G. J. de Jong ${ }^{a}$ \\ Hans J. P. M. Koenen ${ }^{b}$ Ellen H. van den Bogaard ${ }^{a}$ Marieke M. B. Seyger ${ }^{a}$ \\ aDepartment of Dermatology, Radboud University Medical Center, Nijmegen, The Netherlands; ${ }^{b}$ Department \\ of Laboratory Medicine, Laboratory Medical Immunology, Radboud University Medical Center, Nijmegen, \\ The Netherlands; ${ }^{C}$ FibroTx LLC, Tallinn, Estonia; ${ }^{d}$ Department of Chemistry and Biotechnology, Tallinn University \\ of Technology, Tallinn, Estonia
}

\section{Keywords}

Cytokine $\cdot$ Noninvasive $\cdot$ Stratum corneum $\cdot$ Children .

Transdermal analysis patch

\begin{abstract}
Introduction: Transdermal analysis patches (TAPs) noninvasively measure soluble proteins in the stratum corneum. UItimately, such local protein profiles could benefit the search for biomarkers to improve personalized treatment in psoriasis. This study aimed to explore the patient friendliness and protein detection by TAP in pediatric psoriasis in daily clinical practice. Methods: In this observational study, TAPs measuring CXC chemokine ligand (CXCL)-1/2, CC chemokine ligand (CCL)-27, interleukin (IL)-1RA, IL-23, IL-1 a, IL-8, IL-4, IL22, IL-17A, vascular endothelial growth factor (VEGF), human beta-defensin (hBD)-2, hBD-1, and kallikrein-related peptidase (KLK)- 5 were applied on lesional, peri-lesional, and nonlesional skin sites of psoriasis patients aged $>5$ to $<18$ years. Discomfort during TAP removal as an indicator for patient friendliness was assessed by visual analogue scale (VAS;
\end{abstract}

range 0 -10). Results: Thirty-two patients (median age 14.0 years) were included, of which 19 were treated with solely topical agents and 13 with systemic treatment. The median VAS of discomfort during TAP removal was 1.0 (interquartile range 1.0). Significantly higher levels in lesional versus nonlesional skin were found for IL-1RA, VEGF, CXCL-1/2, hBD-2, and IL-8, whereas lower levels were found for IL-1a. Skin surface proteins were measured in both treatment groups, with significant higher lesional levels of KLK-5, IL-1RA, hBD-2, IL$1 \mathrm{a}$, IL-23, and CCL-27 in the systemic treatment group. Conclusion: The TAP platform holds the potential for patientfriendly and noninvasive monitoring of skin-derived proteins in pediatric psoriasis patients in daily clinical practice.

(c) 2021 The Author(s).

Published by S. Karger AG, Basel

Mirjam J. Schaap and Finola M. Bruins contributed equally to the work and share the first authorship.

Ellen H. van den Bogaard and Marieke M.B. Seyger contributed equally.

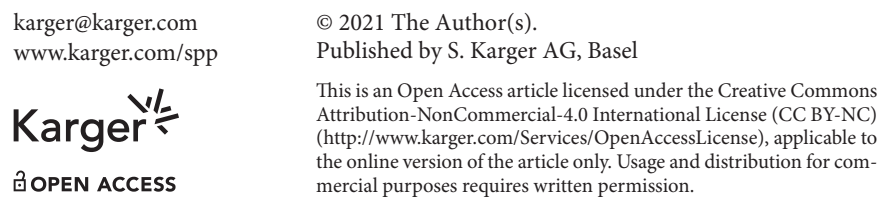

karger@karger.com www.karger.com/spp

Karger ${ }^{\prime \prime}$ -

BOPEN ACCESS

(c) 2021 The Author(s)

Published by S. Karger AG, Basel

This is an Open Access article licensed under the Creative Commons Attribution-NonCommercial-4.0 International License (CC BY-NC) (http://www.karger.com/Services/OpenAccessLicense), applicable to the online version of the article only. Usage and distribution for commercial purposes requires written permission.

Correspondence to:

Mirjam J. Schaap, mirjam.schaap@ radboudumc.nl 


\section{Introduction}

In daily clinical psoriasis care, more guidance regarding treatment response and disease course is desirable. Predictors for disease course are scarce and mainly include clinical patient characteristics (e.g., nail psoriasis and psoriatic arthritis) [1,2]. Research focusing on prediction of treatment success in psoriasis mainly targets the body mass index, genotyping, human leukocyte antigen typing, or blood-derived systemic inflammation markers [3-7]. Besides clinical biomarkers or bloodderived biomarkers, the skin itself provides a unique source of potential biomarkers, like disease-associated profiles of cytokines, chemokines, growth factors, and/or antimicrobial peptides $[3,8,9]$.

Reported protein detection in the psoriatic skin is mostly derived from punch biopsies, an invasive procedure less suitable for longitudinal follow-up with a risk for pain, scarring, and infection [10-14]. Several noninvasive or minimally invasive methods for protein detection or gene expression profiling in the skin exist. Besides the widely used application of repetitive tape stripping to study the skin barrier function and regeneration mechanisms $[15,16]$, tape stripping is used as a sampling method for protein detection [17-20]. In addition, skin lavage, abrasion methods, reverse iontophoresis, and hair root sampling are described [11, 21-23]. Furthermore, one microdisk-library array patch method has been used in a pilot study with 3 healthy adult volunteers [24]. The transdermal analysis patch (TAP), developed by FibroTx, is a new diagnostic tool which noninvasively quantifies proteins directly on the skin surface via spot-enzymelinked immunosorbent assays [25]. Especially in the pediatric patient population, noninvasive methods are key to avoid discomfort and fear for interventions. Additionally, there is a great demand for biomarkers in this specific population considering the chronic aspect of the disease and need for long-term care. In previous research, TAP has shown the potential to monitor dynamic changes in the skin resulting from skin irritating models in healthy individuals [26]. Furthermore, a promising correlation of skin surface proteins sampled by the FibroTx Patch compared to tape strip samples and serum samples was shown in ten adults with atopic dermatitis [27].

In this explorative observational study, we applied the TAP in daily clinical practice in pediatric psoriasis patients. More specifically we assessed (i) the patient friendliness of TAP, (ii) if protein detection is similar on different body locations, (iii) the TAP detection capability of inflammation-related proteins in lesional, peri-lesional, and non-lesional skin, and (iv) whether topical and/or systemic treatment affects the TAP protein detection capability.

\section{Materials and Methods}

Study Design and Population

This study was an explorative observational noninvasive study with internal control in a daily clinical practice setting. Participants were recruited at the outpatient clinic of the Department of Dermatology at the Radboud university medical center in Nijmegen, the Netherlands, between June 2018 and July 2019. Written informed consent and/or assent was given by all subjects and/or their legal guardian before enrollment according to applicable rules. Inclusion criteria were: age $>5$ and $<18$ years, diagnosis of psoriasis according to a dermatologist, enough psoriasis plaques to apply the TAPs at the time of enrollment, and willingness to participate. Patients with another concurrent inflammatory skin disease were excluded. As study procedures occurred in a daily clinical practice setting, patients were already on a topical or systemic treatment at the moment of inclusion. There was no treatment washout phase. The study protocol was approved by the Ethics Committee of the Radboud university medical center (NL60952.091.17).

\section{Transdermal Analysis Patch}

TAP, produced and commercialized by FibroTx, consists of a multiplex capture antibody microarray supported by an adhesive bandage for easy fixture to the skin (see online suppl. Fig. 1; see www.karger.com/doi/10.1159/000516110 for all online suppl. material) [25]. One TAP captures antibody microarray measures up to 7 proteins. The used panel was preset and consists of general skin inflammation-related proteins, of which the majority are classified as chemokines, cytokines, and antimicrobial peptides. More specifically, the following 13 proteins were analyzed by 2 independent TAPs: CXC chemokine ligand (CXCL)-1/2, CC chemokine ligand (CCL)-27, interleukin (IL)-1RA, IL-23, IL-1 $\alpha$, IL-8, IL-4, IL-22, IL-17A, vascular endothelial growth factor (VEGF), human beta-defensin (hBD)-2, hBD-1, and kallikrein-related peptidase (KLK)-5.

\section{Study Procedures}

Patients were not allowed to use any topical medications or toiletries on the investigated sites on the day of the visit. TAPs were applied on plaques located on the arms, legs, or trunk. Lesional skin, peri-lesional skin, and non-lesional skin, with no psoriasis lesions within a distance of $10 \mathrm{~cm}$, were subjected for analysis. To explore the influence of the body location on the protein profile, 2 psoriasis lesions of interest in each patient were chosen preferably on different body parts. To be able to compare protein levels between lesional, peri-lesional, and non-lesional skin, TAPs were also applied peri-lesional of the 2 lesions of interest (online suppl. Fig. 1). After application to the skin, 4 drops of buffer (phosphate buffered saline ( $\mathrm{pH}$ 7.4) as supplied by manufacturer) were added to the reservoir covering the microarray. During the $20 \mathrm{~min}$ application time, antibodies printed on the microarray were allowed to capture skin-derived proteins through immune recognition. After removal, the TAPs were stored at $-20^{\circ} \mathrm{C}$ until the captured pro- 
Table 1. Patient characteristics

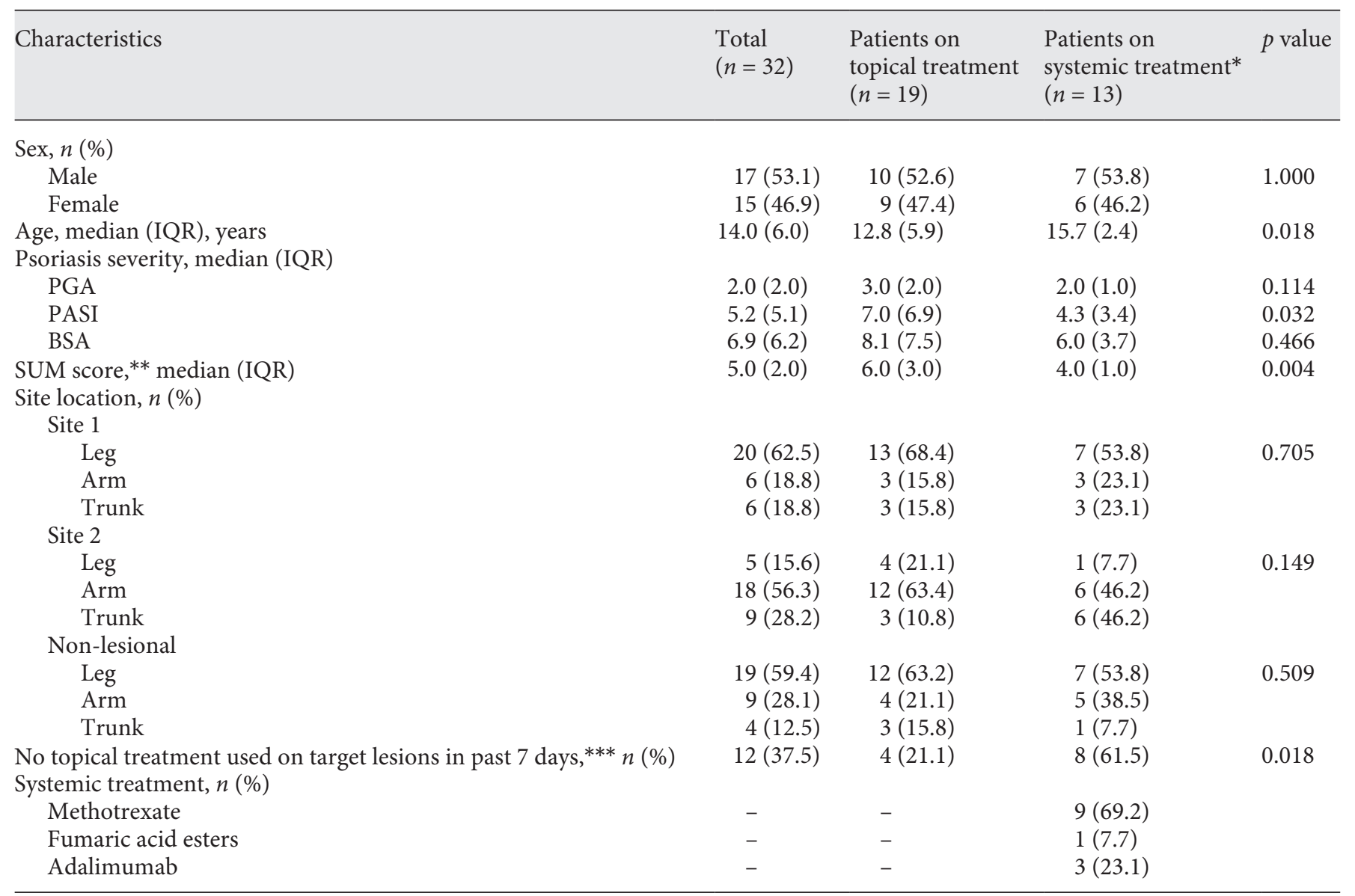

PGA, physician global assessment; PASI, psoriasis area and severity index; BSA, body surface area; SUM score, the sum of the severity for erythema (0-4), induration (0-4) and desquamation (0-4); IQR, interquartile range. * Patients may also use topical treatments (corticosteroids and/or vitamin D derivatives). ${ }^{* *}$ All lesions of both sites combined resulting in a total of 64 lesions ( 2 lesions per patient). *** Topical corticosteroids and/or vitamin $\mathrm{D}$ derivatives.

teins were analyzed using spot-enzyme-linked immunosorbent assay. Values outside the detection levels were regarded as not detectable. Detection levels are described in online suppl. Table 1. All TAPs were applied by 2 physicians (M.J.S. or F.M.B.).

\section{Data Collection}

After removal of the TAPs, experienced discomfort was rated by the subject on a 10-point visual analogue scale (VAS) as an indicator for patient friendliness. A VAS score of 0 meant no discomfort corresponding with a good patient friendliness and a score of 10 meant maximal discomfort. To assess the overall psoriasis severity, the psoriasis area and severity index (PASI; range $0-72$ ), physician global assessment (PGA; range $0-5$ ), and affected body surface area, with higher scores indicating more severe psoriasis, were collected. Further, a SUM score (0-12), defined as the sum of the severity scores for erythema (0-4), induration (0-4), and desquamation (0-4), was calculated in order to determine the severity of the psoriasis lesions sampled by TAPs. Additional collected data included age, sex, cur- rent treatment, and whether or not topical treatments (excluding emollients) were used on the target lesions in the past 7 days.

\section{Statistical Analysis}

Demographic data were presented as numbers (percentage) for categorical variables and medians (interquartile ranges [IQR]) for continuous variables. Protein concentrations were depicted in 2 dimensions based on principal component analysis (PCA). MannWhitney $U$ tests were performed to further assess differences in protein levels. To assess if protein detection was similar on different body locations (inter-donor variability), protein levels on the 2 different sites were compared by the Wilcoxon signed-ranked test for both lesional and peri-lesional measurements. A sensitivity analysis with confounder correction was additionally performed and described in the online suppl. material. Statistical package SPSS, version 25 (IBM), and the statistical programming language $\mathrm{R}$ version 3.6.2 were used to perform analyses. A twosided $p<0.05$ was considered statistically significant. 
Fig. 1. PCA of protein levels in lesional ( $n=64$ samples), peri-lesional ( $n=64$ samples), and non-lesional skin ( $n=32$ samples). Biplot of dimension 1 (horizontal axis) and 2 (vertical axis). Each dot represents one sample. Levels of 11 proteins were included: CXCL-1/2, CCL-27, IL1RA, IL-23, IL-1a, IL-8, IL-22, VEGF, hBD-2, hBD-1, and KLK-5. CXCL, CXC chemokine ligand; CCL, CC chemokine ligand; IL, interleukin; VEGF, vascular endothelial growth factor; hBD, human betadefensin; KLK, kallikrein-related peptidase; PCA, principal component analysis.

\section{Results}

\section{Patient Characteristics}

In total, 32 patients were included with a median age of 14.0 (IQR 6.0) years and a median PASI score of 5.2 (IQR 5.1) (Table 1). At the time of measurements, solely topical agents were used by 19 patients, while 13 patients were on systemic therapy, with concomitant use of topical treatments if required. Patients on systemic therapy were treated with methotrexate $(n=9)$, fumaric acid esters $(n=1)$, or adalimumab $(n=3)$. Overall, $12(37.5 \%)$ patients did not use any topical treatments on the target lesions 7 days prior to the TAP applications, of which 8
(61.5\%) were from the systemic treatment group. Patients on systemic therapy had significantly lower PASI scores and were significantly older compared to patients on topical agents (Table 1). One patient mentioned a burning sensation after removal of the TAP, no other adverse events were reported. The median reported VAS score for discomfort during removal was 1.0 (IQR 1.0).

\section{Protein Profiles of Lesional and Peri-Lesional Skin Are Similar on Different Sites}

Comparison of the 2 different skin sites within 1 patient with the Wilcoxon signed-ranked test showed no significant differences for each individual protein on the 


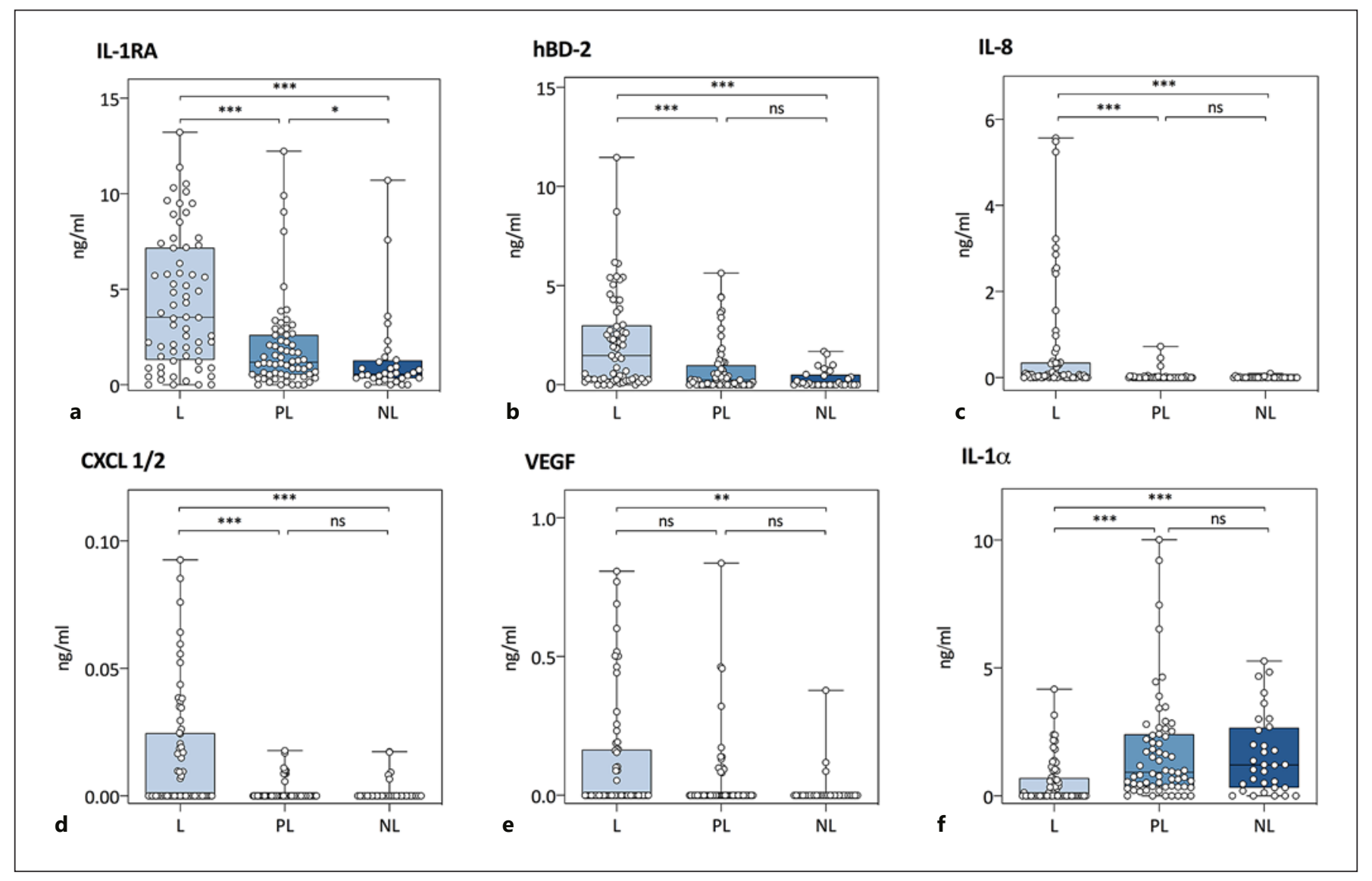

Fig. 2. Absolute counts of proteins measured in lesional ( $n=64$ samples), peri-lesional ( $n=64$ samples), and non-lesional ( $n=32$ samples) skin by TAP. Each dot represents one measurement. The Mann-Whitney U test was used to assess differences between (i) lesional and peri-lesional skin, (ii) peri-lesional and non-lesional skin, and (iii) lesional and non-lesional skin. L, lesional; PL, peri-lesional; NL, non-lesional; ${ }^{*} p<0.05,{ }^{* *} p<0.01$, $* * * p<0.001$; ns, nonsignificant; CXCL, CXC chemokine ligand; IL, interleukin; VEGF, vascular endothelial growth factor; hBD, human beta-defensin; TAP, transdermal analysis patch.

group level (online suppl. Table 1,2). Therefore, since significant differences between different (body) sites were not found, and to improve power, measurements were combined for further analyses, resulting in 64 lesional and 64 peri-lesional samples. IL-4 and IL-17A were excluded from analyses as only one (IL-4) and no measurements (IL-17A) were above the lower detection level.

TAP Detects Different Protein Levels in Lesional, PeriLesional, and Non-Lesional Skin

To explore the combinatory effect of the protein profile in lesional versus peri-lesional and non-lesional skin, a nonmetric multidimensional scaling approach, that is, PCA analysis, was used. We revealed a separation between nonlesional, peri-lesional, and lesional samples in the first dimension (Fig. 1). In line with known psoriasis-related ex- pression profiles, further comparison per protein shows significantly higher levels for IL-1RA, hBD-2, IL-8, and CXCL-1/2 in the lesional skin compared to both nonlesional and peri-lesional skin (Fig. 2; online suppl. Table 4) $[17,18]$. VEGF showed a significantly higher level for the lesional skin compared to non-lesional skin, but not compared to peri-lesional skin. In contrast, significantly lower protein levels were found for IL-1 $\alpha$ in the lesional skin compared to both peri-lesional and non-lesional skin, which corresponds with previously reported research [18]. No significant differences between lesional, peri-lesional, and non-lesional skin were detected for IL-23, CCL-27, IL-22, hBD-1, and KLK-5, although a trend for higher levels of IL23 and IL-22 in lesional skin compared to non-lesional skin was observed. A sensitivity analysis with confounder correction (for sex, age, body site, treatment, use of topical cor- 
Fig. 3. PCA of protein levels in the lesional skin ( $n=64$ samples) split for measurements of patients on solely topical treatment ( $n=38$ samples) versus systemic treatment ( $n=26$ samples). Biplot of dimension 1 (horizontal axis) and 2 (vertical axis). Each dot represents 1 sample. Levels of 11 proteins were included: CXCL-1/2, CCL-27, IL-1RA, IL-23, IL-1 $\alpha$, IL-8, IL-22, VEGF, hBD-2, hBD-1, and KLK-5. CXCL, CXC chemokine ligand; CCL, CC chemokine ligand; IL, interleukin; VEGF, vascular endothelial growth factor; hBD, human beta-defensin; KLK, kallikrein-related peptidase; PCA, principal component analysis.

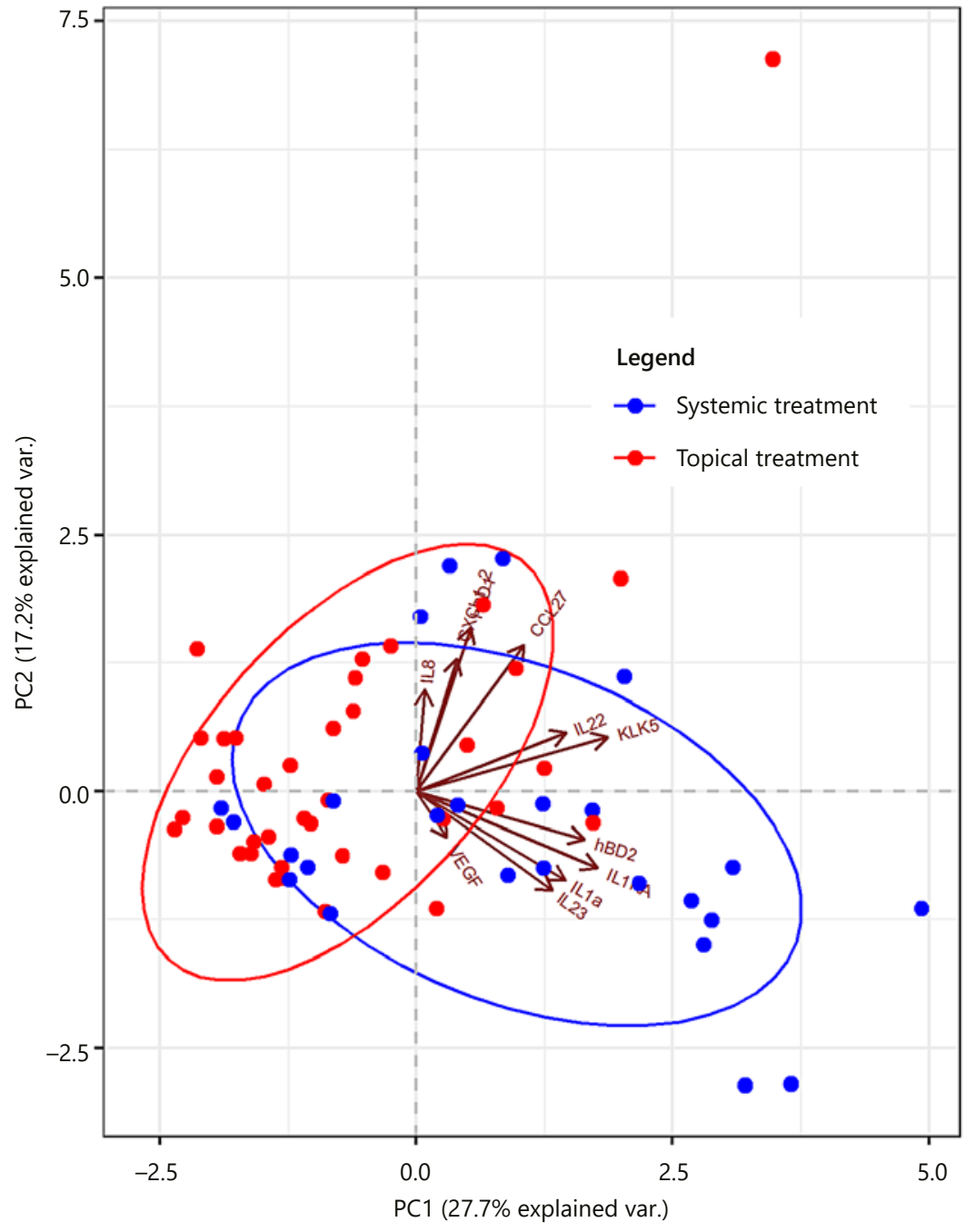

ticosteroids in the last 7 days, and PGA) revealed similar results (online suppl. Table 5).

\section{Protein Detection in Lesional Skin during Therapeutic Regimens}

As both patients on topical and on systemic treatments were included in this daily clinical practice study, the possible influence of topical and systemic treatments on the TAP protein detection was explored. PCA showed that proteins detected in the lesional skin of patients on systemic treatment are separated from those derived from patients solely on topical treatment (Fig. 3). A detailed comparison between treatment groups per protein is shown in Figure 4 and online suppl. Table 6. In patients on systemic treatment ( $n=26$ samples), significantly higher levels of IL-1 $\alpha$, IL-1RA, IL-23, hBD-2, CCL-27, and KLK-5 were measured compared to solely topically treated patients ( $n=38$ samples). After a sensitivity analysis with confounder correction (for sex, age, body site, use of topical corticosteroids on investigated lesion in last 7 days, a SUM score, and PGA), levels of IL-1RA, IL- $1 \alpha$, and IL-23 remained higher in the lesional skin of patients on systemic treatment (online suppl. Table 7).

\section{Discussion}

In our study, several key elements for the clinical applicability of TAP were identified. First, the finding that pediatric patients experienced almost no discomfort dur- 


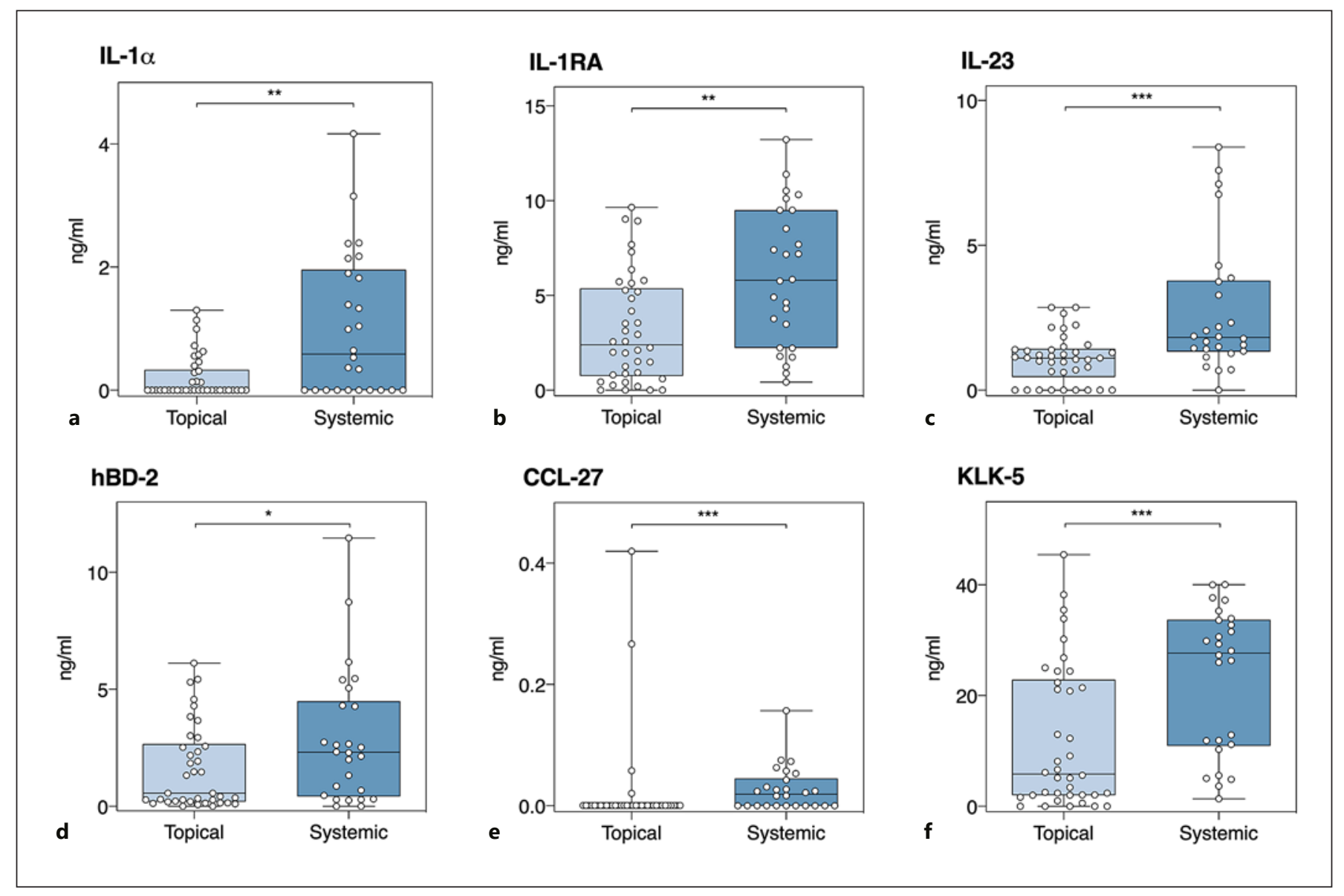

Fig. 4. Absolute counts of proteins in the lesional skin ( $n=64$ samples) measured by TAP split for patients on solely topical treatment ( $n=38$ samples) versus systemic treatment ( $n=26$ samples). Each dot represents 1 measurement. The Mann-Whitney U test was used to assess differences between measurements of patients on solely topical versus systemic treatment. L, lesional; PL, peri-lesional; NL, non-lesional; ${ }^{*} p<0.05,{ }^{* *} p<0.01,{ }^{* * *} p<$ 0.001; ns, nonsignificant. CCL, CC chemokine ligand; IL, interleukin; hBD, human beta-defensin; KLK, kallikrein-related peptidase; TAP, transdermal analysis patch.

ing application and removal of the TAPs supports the use of TAPs in daily clinical practice. Second, no prominent differences in protein profiles of (peri-)lesional skin were seen between different (body) sites. Although larger studies are required to confirm this, these results suggest the potential applicability of TAP on any desired (body) site. Third, both in patients on solely topical and systemic treatment, we were able to detect significant differences in protein levels between lesional and non-lesional skin, which increases the applicability of TAP in daily clinical practice. Finally, in contrast to repetitive tape stripping, TAP collects proteins from an intact stratum corneum, which reduces the risk for local skin irritation and therefore decreases the risk of koebnerization for psoriasis patients. A reduced risk for local skin irritation might also have implications for the implementation of TAPs for the monitoring of other inflammatory skin conditions with a poor barrier function, such as atopic dermatitis.

Recent studies on noninvasive skin sampling for the analysis of local skin disease signatures underscore the demand and importance of such new sampling methodologies and analyses $[19,20]$. This paradigm shift towards patient friendly, high end molecular disease profiling provides an alternative to the conventional use of invasive skin biopsies. Especially for the pediatric patient population these developments are of utmost importance. Similar to the RNA [20] and proteome profiling using tape strips [19] in psoriasis and atopic dermatitis, respectively, our data using TAP technology illustrates the potential of noninvasive localized sampling for quantitative investi- 
gations. The potential of the skin surface protein measurement by TAP is further underlined by a recent study in 10 adult atopic dermatitis patients, which revealed a promising correlation between the skin surface proteins sampled by the FibroTx Patch, tape strip skin samples, and serum samples [27].

Although no proteome analysis studies in pediatric psoriasis patients are reported, in line with previous proteome analysis using tape stripping in adult psoriasis patients, we found higher levels of VEGF, CXCL-1/2, IL-8, and IL-23 and lower levels of IL- $1 \alpha$ in the lesional skin $[17,18]$. The role of these general skin inflammatory proteins includes neutrophil infiltration and Th17-cell differentiation [18]. The reproducibility of our findings indicates the robustness of TAP for the skin surface protein detection. The proteins we could include in our analysis were mostly keratinocyte-derived and enabled the differentiation between lesional and non-lesional skin. Even more subtle differences after confounder correction between non-lesional and peri-lesional skin were highlighted by the highly expressed psoriasis-associated epidermal marker protein, hBD-2 (online suppl. Table 5), whose tissue and serum levels are known to correlate well to disease severity [3]. Immune cell-derived proteins IL-4 and IL-17A were excluded from analyses as only one (IL-4) and no measurements (IL-17A) exceeded the minimum detection level. According to the known psoriasis pathogenesis, it is expected that IL- 4 would not be detected in the skin surface. Instead, IL-17A was anticipated to be detected being a key pro-inflammatory cytokine in psoriasis. Furthermore, IL-17A detection is described in previous tape stripping studies [18]. In our study, IL-17A was measured in 15 out of 64 lesional samples, albeit values did not meet the minimum detection level. Current detection limits of the TAPs might not be sensitive enough to sufficiently measure soluble immune cell-derived proteins via diffusion through the stratum corneum, which might explain why IL-17A was not widely detected in our study.

We showed that TAP is capable to detect soluble skin proteins in patients receiving topical-and systemic treatments. Intriguingly, statistically significant lower protein levels in the lesional skin of patients on solely topicals versus those on systemic therapy were found. Even after sensitivity analysis with correction for the use of topical treatments in the last 7 days and for other possible confounders (PGA, SUM, age, and gender) with a linear mixed model, levels of IL-1RA, IL-1 $\alpha$, and IL-23 remained significantly higher in patients on systemic therapy (online suppl. Table 7). It has to be noted that our study de- sign was explorative and the study was performed in daily clinical practice. Therefore, given the heterogeneity of our population, conclusions regarding the biological relevance of these differences are difficult to draw and would require a specifically designed follow-up study. However, one could hypothesize that the difference in protein detection in treatment groups is due to the direct negative influence of chronic and repetitive application of topical agents on protein levels in the stratum corneum. To explore this hypothesis, we also compared proteins between treatment groups on non-lesional skin sites, which were never treated with topical agents irrespective of the treatment group. Intriguingly, also in non-lesional skin, significantly higher levels of KLK-5, IL-1RA, IL-23, and IL$1 a$ were seen in patients treated with systemic agents (online suppl. Table 6). Experimental evidence to substantiate these findings should be gained from future studies in patient cohorts with controlled treatment regimens.

Although we ultimately search for implementation of TAP in daily clinical practice, there is still a long road ahead. Further research is needed to determine the optimal marker set for the application of TAP in (pediatric) psoriasis. Given the explorative nature of this study, a relatively small number of patients was included. It also has to be noted that the marker set captured from the skin surface was a general inflammatory preset protein panel, thus not specific for psoriasis. However, for future studies, the protein panel could be adjusted according to disease specific signature proteins, enabling the use of TAP in other inflammatory skin diseases. Our data are based on protein measurements at one time point. Longitudinal follow-up data of the present clinical practice cohort would increase insights into the consistency of the protein profile present in the skin in each individual. This could enable an understanding of the correlation with disease course and treatment response. Acquiring disease- or patient-specific proteins directly from the skin surface by using TAP could enhance mapping of underlying skin disease mechanisms, personalized treatment, and ultimate prediction of disease course.

\section{Conclusion}

Protein detection in the stratum corneum of the skin by TAP is regarded patient friendly in pediatric psoriasis patients. TAP noninvasively measures proteins directly from the skin regardless of treatment and provides a promising platform for implementation of protein collection in clinical practice or research. 


\section{Statement of Ethics}

This research was conducted in accordance with the World Medical Association Declaration of Helsinki. Written informed consent and/or assent was given by all subjects and/or their legal guardian before enrollment according to applicable rules. The study protocol was approved by the Ethics Committee of the Radboud university medical center (NL60952.091.17).

\section{Conflict of Interest Statement}

M.J. Schaap has carried out clinical trials for Amgen, Celgene, Janssen, and Lilly. F.M. Bruins has carried out clinical trials for Abbvie, Amgen, Celgene, Janssen, Leo Pharma, Lilly, and Pfizer. $\mathrm{X}$. He has no conflicts of interest. K. Orro is employed by FibroTx. M. Peppelman has no conflicts of interest. P.E.J. van Erp has no conflicts of interest. E.M.G.J. de Jong has received research grants for the independent research fund of the department of dermatology of the Radboud university medical center Nijmegen, The Netherlands from AbbVie, Novartis, Janssen Pharmaceutica, and Leo Pharma and has acted as consultant and/or paid speaker for and/or participated in research sponsored by companies that manufacture drugs used for the treatment of psoriasis including AbbVie, Janssen Pharmaceutica, Novartis, Lily, Celgene, Leo Pharma, UCB, and Almirall. All funding is not personal but goes to the independent research fund of the department of dermatology of Radboud university medical center Nijmegen, The Nether- lands. H.J.P.M. Koenen has no conflicts of interest. E.H. van den Bogaard has no conflicts of interest. M.M.B. Seyger received grants from/was involved in clinical trials from Abbvie, Amgen, Celgene, Eli Lilly, Janssen, Leo Pharma, and Pfizer. She served as a consultant for Abbvie, Eli Lilly, Janssen, Leo Pharma, Novartis, UCB, and Pfizer; fees were paid directly to the institution.

\section{Funding Sources}

This study was funded by Innovatiefonds zorgverzekeraars. TAPs were provided by FibroTx.

\section{Author Contributions}

Mirjam J. Schaap and Finola. M. Bruins: contributed to data acquisition, data analysis, interpretation of data, and wrote the manuscript. Xuehui He: contributed to data analysis, interpretation of data, and wrote the manuscript. Kadri Orro: contributed to research design, data analysis, interpretation of data, and manuscript revisions. Malou Peppelman, Piet E.J. van Erp, and Elke M.G.J. de Jong: contributed to research design and manuscript revisions. Hans J.P.M. Koenen: contributed to interpretation of data and manuscript revisions. Ellen H. van den Bogaard and Marieke M.B. Seyger: contributed to research design, interpretation of data, and manuscript revisions.

\section{References}

1 Raposo I, Torres T. Nail psoriasis as a predictor of the development of psoriatic arthritis. Actas Dermosifiliogr. 2015 Jul-Aug; 106(6): 452-7.

2 Bronckers IMGJ, Bruins FM, van Geel MJ, Groenewoud HMM, Kievit W, van de Kerkhof PCM, et al. Nail involvement as a predictor of disease severity in paediatric psoriasis: follow-up data from the dutch ChildCAPTURE registry. Acta Derm Venereol. 2019 Feb 1;99(2):152-7.

3 Jansen PA, Rodijk-Olthuis D, Hollox EJ, Kamsteeg M, Tjabringa GS, de Jongh GJ, et al. Beta-defensin-2 protein is a serum biomarker for disease activity in psoriasis and reaches biologically relevant concentrations in lesional skin. PLoS One. 2009;4(3):e4725.

4 Singh S, Facciorusso A, Singh AG, Vande Casteele N, Zarrinpar A, Prokop LJ, et al. Obesity and response to anti-tumor necrosis factor-alpha agents in patients with select immune-mediated inflammatory diseases: a systematic review and meta-analysis. PLoS One. 2018;13(5): 0195123.

5 Solberg SM, Sandvik LF, Eidsheim M, Jonsson R, Bryceson YT, Appel S. Serum cytokine measurements and biological therapy of psoriasis: prospects for personalized treatment? Scand J Immunol. 2018 Dec;88(6):e12725.
6 Dand N, Duckworth M, Baudry D, Russell A, Curtis CJ, Lee SH, et al. HLA-C*06:02 genotype is a predictive biomarker of biologic treatment response in psoriasis. J Allergy Clin Immunol. 2019 Jun;143(6):2120-30.

7 van Vugt LJ, van den Reek JMPA, Meulewaeter E, Hakobjan M, Heddes N, Traks T, et al. Response to IL-17A inhibitors secukinum$a b$ and ixekizumab cannot be explained by genetic variation in the protein-coding and untranslated regions of the IL-17A gene: results from a multicentre study of four European psoriasis cohorts. J Eur Acad Dermatol Venereol. 2020 Jan;34(1):112-8.

8 Baliwag J, Barnes DH, Johnston A. Cytokines in psoriasis. Cytokine. 2015 Jun;73(2):34250.

9 Chularojanamontri L, Charoenpipatsin N, Silpa-Archa N, Wongpraparut C, Thongboonkerd V. Proteomics in psoriasis. Int J Mol Sci. 2019 Mar 6;20(5).

10 Li J, Chen X, Liu Z, Yue Q, Liu H. Expression of Th17 cytokines in skin lesions of patients with psoriasis. J Huazhong Univ Sci Technol Med Sci. 2007 Jun;27(3):330-2.

11 Wang CY, Maibach HI. Why minimally invasive skin sampling techniques? A bright scientific future. Cutan Ocul Toxicol. 2011 Mar; 30(1):1-6.
12 Swindell WR, Remmer HA, Sarkar MK, Xing X, Barnes DH, Wolterink L, et al. Proteogenomic analysis of psoriasis reveals discordant and concordant changes in mRNA and protein abundance. Genome Med. 2015;7(1):86.

13 Cordoro KM, Hitraya-Low M, Taravati K, Sandoval PM, Kim E, Sugarman J, et al. Skininfiltrating, interleukin-22-producing $\mathrm{T}$ cells differentiate pediatric psoriasis from adult psoriasis. J Am Acad Dermatol. 2017 Sept; 77(3):417-24.

14 Kolbinger F, Loesche C, Valentin MA, Jiang $\mathrm{X}$, Cheng $\mathrm{Y}$, Jarvis $\mathrm{P}$, et al. $\beta$-Defensin 2 is a responsive biomarker of IL-17A-driven skin pathology in patients with psoriasis. J Allergy Clin Immunol. 2017 Mar;139(3):923-e8.

15 Dickel H, Goulioumis A, Gambichler T, Fluhr JW, Kamphowe J, Altmeyer P, et al. Standardized tape stripping: a practical and reproducible protocol to uniformly reduce the stratum corneum. Skin Pharmacol Physiol. 2010; 23(5):259-65.

16 Pfannes EKB, Weiss L, Hadam S, Gonnet J, Combardière B, Blume-Peytavi U, et al. Physiological and molecular effects of in vivo and ex vivo mild skin barrier disruption. Skin Pharmacol Physiol. 2018;31(3):115-24. 
17 Benson NR, Papenfuss J, Wong R, Motaal A, Tran V, Panko J, et al. An analysis of select pathogenic messages in lesional and non-lesional psoriatic skin using non-invasive tape harvesting. J Invest Dermatol. 2006 Oct; 126(10):2234-41.

18 Mehul B, Laffet G, Seraidaris A, Russo L, Fogel P, Carlavan I, et al. Noninvasive proteome analysis of psoriatic stratum corneum reflects pathophysiological pathways and is useful for drug profiling. Br J Dermatol. 2017 Aug; 177(2):470-88.

19 Guttman-Yassky E, Diaz A, Pavel AB, Fernandes M, Lefferdink R, Erickson T, et al. Use of tape strips to detect immune and barrier abnormalities in the skin of children with early-onset atopic dermatitis. JAMA Dermatol. 2019 Oct 9;155(12):1358-70.
$20 \mathrm{He} \mathrm{H}$, Bissonnette R, Wu J, Diaz A, Saint-Cyr Proulx E, Maari C, et al. Tape strips detect distinct immune and barrier profiles in atopic dermatitis and psoriasis. J Allergy Clin Immunol. 2021 Jan;147(1):199-212.

21 Aubert J, Reiniche P, Fogel P, Poulin Y, Lui H Lynde C, et al. Gene expression profiling in psoriatic scalp hair follicles: clobetasol propionate shampoo $0.05 \%$ normalizes psoriasis disease markers. J Eur Acad Dermatol Venereol. 2010 Nov;24(11):1304-11.

22 Emson CL, Fitzmaurice S, Lindwall G, Li KW Hellerstein MK, Maibach HI, et al. A pilot study demonstrating a non-invasive method for the measurement of protein turnover in skin disorders: application to psoriasis. Clin Transl Med. 2013;2:12.

23 Portugal-Cohen M, Kohen R. Non-invasive evaluation of skin cytokines secretion: an innovative complementary method for monitoring skin disorders. Methods. 2013 May 15; 61(1):63-8.
24 Oh DY, Na H, Song SW, Kim J, In H, Lee AC, et al. ELIPatch, a thumbnail-size patch with immunospot array for multiplexed protein detection from human skin surface. Biomicrofluidics. 2018 May;12(3):031101.

25 Orro K, Smirnova O, Arshavskaja J, Salk K, Meikas A, Pihelgas S, et al. Development of TAP, a non-invasive test for qualitative and quantitative measurements of biomarkers from the skin surface. Biomark Res. 2014;2: 20.

26 Falcone D, Spee P, Salk K, Peppelman M, van de Kerkhof PCM, van Erp PEJ. Measurement of skin surface biomakers by Transdermal Analyses Patch following different in vivo models of irritation: a pilot study. Skin Res Technol. 2017 Aug;23(3):336-45.

27 Røpke MA, Mekulova A, Pipper C, Eisen M, Pender K, Spee P, et al. Non-invasive assessment of soluble skin surface biomarkers in atopic dermatitis patients - effect of treatment. Skin Res Technol. Epub 2021 Jan 28. 